\title{
Raman spectroscopic investigation of spinal cord injury in a rat model
}

\author{
Tarun Saxena, ${ }^{\mathrm{a}}$ Bin Deng, ${ }^{\mathrm{a}}$ Dennis Stelzner, ${ }^{\mathrm{b}}$ Julie Hasenwinkel, ${ }^{\mathrm{a}}$ and Joseph Chaiken ${ }^{\mathrm{c}}$ \\ ${ }^{a}$ Syracuse University, Department of Biomedical and Chemical Engineering, Syracuse, New York 13244 \\ bSUNY Upstate Medical University, Department of Cell and Developmental Biology, Syracuse, New York 13210 \\ 'CSyracuse University, Department of Chemistry, Syracuse, New York 13244
}

\begin{abstract}
Raman spectroscopy was used to study temporal molecular changes associated with spinal cord injury (SCI) in a rat model. Raman spectra of saline-perfused, injured, and healthy rat spinal cords were obtained and compared. Two injury models, a lateral hemisection and a moderate contusion were investigated. The net fluorescence and the Raman spectra showed clear differences between the injured and healthy spinal cords. Based on extensive histological and biochemical characterization of $\mathrm{SCl}$ available in the literature, these differences were hypothesized to be due to cell death, demyelination, and changes in the extracellular matrix composition, such as increased expression of proteoglycans and hyaluronic acid, at the site of injury where the glial scar forms. Further, analysis of difference spectra indicated the presence of carbonyl containing compounds, hypothesized to be products of lipid peroxidation and acid catalyzed hydrolysis of glycosaminoglycan moieties. These results compared well with in vitro experiments conducted on chondroitin sulfate sugars. Since the glial scar is thought to be a potent biochemical barrier to nerve regeneration, this observation suggests the possibility of using near infrared Raman spectroscopy to study injury progression and explore potential treatments ex vivo, and ultimately monitor potential remedial treatments within the spinal cord in vivo. @ 2011 Society of Photo-Optical Instrumentation Engineers (SPIE). [DOI: 10.1117/1.3549700]
\end{abstract}

Keywords: spinal cord injury; glial scar; Raman spectroscopy; chondroitin sulfate proteoglycans.

Paper 10401PR received Jul. 15, 2010; revised manuscript received Dec. 4, 2010; accepted for publication Jan. 3, 2011; published online Feb. 22, 2011.

\section{Introduction}

Spinal cord injury (SCI) is a debilitating condition, leading to paralysis, and currently affects more than 250,000 Americans with annual healthcare costs exceeding 40 billion dollars. ${ }^{1,2}$ Intensive research efforts are ongoing to understand and treat SCI. Currently, histology and immunohistochemistry are gold standards for obtaining qualitative and quantitative information about cellular and biochemical components of tissues. Immunohistochemical techniques have also been used to characterize the injured spinal cord post-SCI. However, these are exclusively ex vivo techniques, requiring preprocessing of tissues and altering their native state. The main goal of this research was to explore the possible use of Raman spectroscopy (RS) in assessing the chemical and physical state of spinal cord tissue in its native physiological state after SCI.

There are two main types of SCI and they are classified based on the injury mechanism. A contusion injury results when the surface of the spinal cord is bruised due to blunt trauma, such as a blow to the spinal column in an accident. This is the most common type of spinal cord injury seen in the clinic. Injury can also be caused by laceration of the spinal cord when a sharp object pierces the cord, as in a knife or bullet wound. A hemisection injury is a special case of a laceration injury when one lateral half of the spinal cord is cut using a sharp instrument. This kind of injury is less likely in a clinical setting but is useful as an experimental model since the injury is focal and damage

Address all correspondence to: Joseph Chaiken, Syracuse University, Department of Chemistry, Syracuse, New York, 13244. Tel: 315443 4285; E-mail: jchaiken@syr.edu. to the cord is localized to a small region around the site of injury. ${ }^{3,4}$

Irrespective of the type of SCI, the sequence of events following SCI can be divided into three phases: the primary or immediate phase, the secondary or acute phase, and the chronic phase. $^{5,6}$ In the immediate phase, due to the mechanical insult to the spinal cord at the lesion site, there is a complex cascade of primary events followed by secondary effects orchestrated by the host's immune system. There is immediate cell death of neurons and glial cells such as oligodendrocytes and astrocytes, and endothelial cells due to the mechanical trauma locally at the site of injury and the surrounding tissue. Increased neuronal and astrocytic cell death and invasion of inflammatory cells such as macrophages, and monocytes, causes an increase in the concentration of excitatory molecules such as glutamate and peroxides. Further, when astrocytes are subjected to trauma, they become reactive, which causes them to proliferate and increase the production of intermediate filaments made up of glial fibrillary acidic protein. The secondary phase lasts for hours to a few days. During this period astrocytes begin to migrate out of the lesion center, producing molecules such as proteoglycans and laminin in the extracellular space. In the chronic phase of injury there is continued necrosis and also demyelination in the white matter, due to apoptotic oligodendrocytic death. Reactive astrocytes continue to invade the region surrounding the lesion center and begin to wall off this region, forming what is classically known as the glial scar. The glial scar walls off the site of injury and consists mainly of reactive astrocytes and

1083-3668/2011/16(2)/027003/14/\$25.00 (C) 2011 SPIE 
the chondroitin sulfate proteoglycans (CSPGs) secreted by these astrocytes. Further, due to meningeal breakdown in the case of a hemisection injury, there is an infiltration of fibroblasts into the lesion site and these fibroblasts produce collagen. ${ }^{3}$ It is the presence of this glial scar and the lesion core that it subsumes, which is inhibitory to axonal regeneration. The glial scar has been hypothesized to be a physical and a biochemical barrier to axonal regeneration. $3,7,8$

Regenerating axons often retract or stop extending once they encounter the glial scar. The extracellular matrix (ECM) of the glial scar consists of both inhibitory (e.g., CSPGs) and growth permissive molecules (e.g., laminin). Sulfated proteoglycans are a potent class of inhibitory ECM molecules and enzymatic degradation of CSPGs using chondroitinase $\mathrm{ABC}(\mathrm{cABC})$ to remove the glycosaminogylcan (GAG) side chains of the molecule has shown promising results ${ }^{9}$ in terms of improved axonal regeneration, sprouting, and functional recovery.

The consequences of SCI are quite complex, but spontaneous RS generally is not a technique capable of detecting materials in mixtures at less than the part per thousand level of concentration. This has the effect of simplifying the acquired data in the sense that observable Raman spectral features ex vivo are dominated by materials that are present in relatively high concentrations or have repeating units that cause spectral features to accumulate in certain spectral regions, for example amide I or $\mathrm{CH}_{2}$ deformation. Any small molecule analyte present in less than 1 millimolar concentration is not likely to be observed.

The objective of this work was to develop the use of RS as a reliable method to temporally and spatially identify various biochemical components of the glial scar in its native physiological state; without homogenization, extraction, the use of dyes, or contrast enhancing labels. All of the biomolecules that are produced in reaction to SCI are composed of proteins, lipids, and carbohydrates. The linkages between them are the features that vary in the Raman spectra of healthy versus injured cord, in addition to the internal modes of these constituents, which have become well known from the last 30 years of biological spectroscopy. ${ }^{10}$ Further, similar to post-transcriptional and enzymatic and nonenzymatic modifications of proteins in healthy cells and tissues, we expect such processes to also occur in injured spinal cord. It is our hope that successful culmination of this work will begin to provide a sound basis for future in vivo work. The animal model chosen for this work was the rat, with the injury model being either a lateral hemisection or a moderate contusion. The hemisected glial scar was evaluated spectroscopically at four days, two weeks, and eight weeks postinjury. We then investigated moderately contused spinal cords, two weeks post-injury. We also conducted in vitro experiments on chondroitin sulfate (CS) polysaccharide solutions by monitoring changes in Raman spectra of CS solutions upon addition of $\mathrm{cABC}$.

\section{Methodology}

\subsection{Hemisection and Contusion Injury}

The Department of Laboratory Animal Resources and the Committee for the Humane Use of Animals at SUNY Upstate Medical University approved this study following Association for Assessment and Accreditation of Laboratory Animal Care guidelines. Adult, female Sprague-Dawley rats (average weight
255 gram) were used for all experiments. Rats were anesthetized with Ketamine and Xylazine ( 80 and $10 \mathrm{mg} / \mathrm{kg}$, respectively) and body temperature was maintained using a heating pad. Aseptic surgery was performed using the following technique. The skin over the upper thoracic area was shaved and cleaned with a Betadine solution. The skin was incised, and then the connective and muscle tissue were bluntly dissected to expose the thoracic ninth (T9) vertebral body. A T9 laminectomy was completed, taking care not to damage the spinal cord during removal of the dorsal lamina. A lateral hemisection was performed at T9; initially a surgical needle punctured the spinal cord dorsoventrally at the midline avoiding the dorsal spinal artery; angled microscissors were then used to cut the right half of the spinal cord followed by an angled needle scraping the vertebrae ventrally and laterally surrounding the lesion to ensure completeness of the hemisection. Finally, the lesion was closed in layers with individual sutures. A schematic of the hemisection surgery is shown in Fig. 1. For the contusion injury, rats were surgically prepared as described. After exposing the spinal cord at T9, animals were injured using the Infinite Horizons (IH) impaction device (Precision Systems and Instrumentation, LLC). The details of the method of operation of the IH impactor can be found elsewhere. ${ }^{11}$ Briefly, a contusion injury to the exposed spinal cord was achieved by rapidly applying a force-defined impact with a stainless steel-tipped impounder. The impounder, controlled by a stepping motor, applied a user-defined force to the spinal cord (200 kDyne). This load has previously been known to produce a moderate injury. ${ }^{6,11}$

The animals were sacrificed at various time points, postinjury. Injured spinal cords were excised using a posterior approach. Euthanasia, by perfusion of deeply anaesthetized rats with physiological phosphate buffered saline (PBS), was followed by a laminectomy between the first cervical vertebra $(\mathrm{C} 1)$ and the fourth lumbar vertebra (L4). The nerve roots were carefully severed and the spinal cord was cut at levels C1 and L4, then carefully removed and placed in a bath of isotonic PBS. No attempt was made to remove the dura mater. The cords were then stored in a refrigerator at $4^{\circ} \mathrm{C}$ until testing. All testing was performed within $4 \mathrm{~h}$ of excision of the spinal cords. A total of 30 animals were used. Six animals were used for each time point post-injury for the hemisection model ( $n=18$ total). Six animals were used for the contusion group, and sacrificed, two weeks post-SCI, and six animals were used as controls. The control animals did not receive any injury and the excised spinal cords were termed as healthy. Two spinal cords from the 4 day post-injury group were not used due to (a) the laser burning the cord due to excess blood and (b) one cord was too fragile and proved difficult to excise in its entirety.

\subsection{Chondroitin Sulfate Solutions and the Effect of $C A B C$}

Chondroitin 6-sulfate (CS6, Sigma Aldrich) was used for in vitro experiments. The glycosaminoglycan powder was used as received. To study the effect of cABC (Seikagaku Corporation) on CS6, $100 \mu \mathrm{l}$ of cABC solution (2 Units/ml) was added to a $5 \mathrm{ml}$ solution of $5 \mathrm{mg} / \mathrm{ml} \mathrm{CS6}$ at room temperature and spectra were collected in a specially designed quartz cuvette at 20-min intervals for a period of $1 \mathrm{~h}$. 

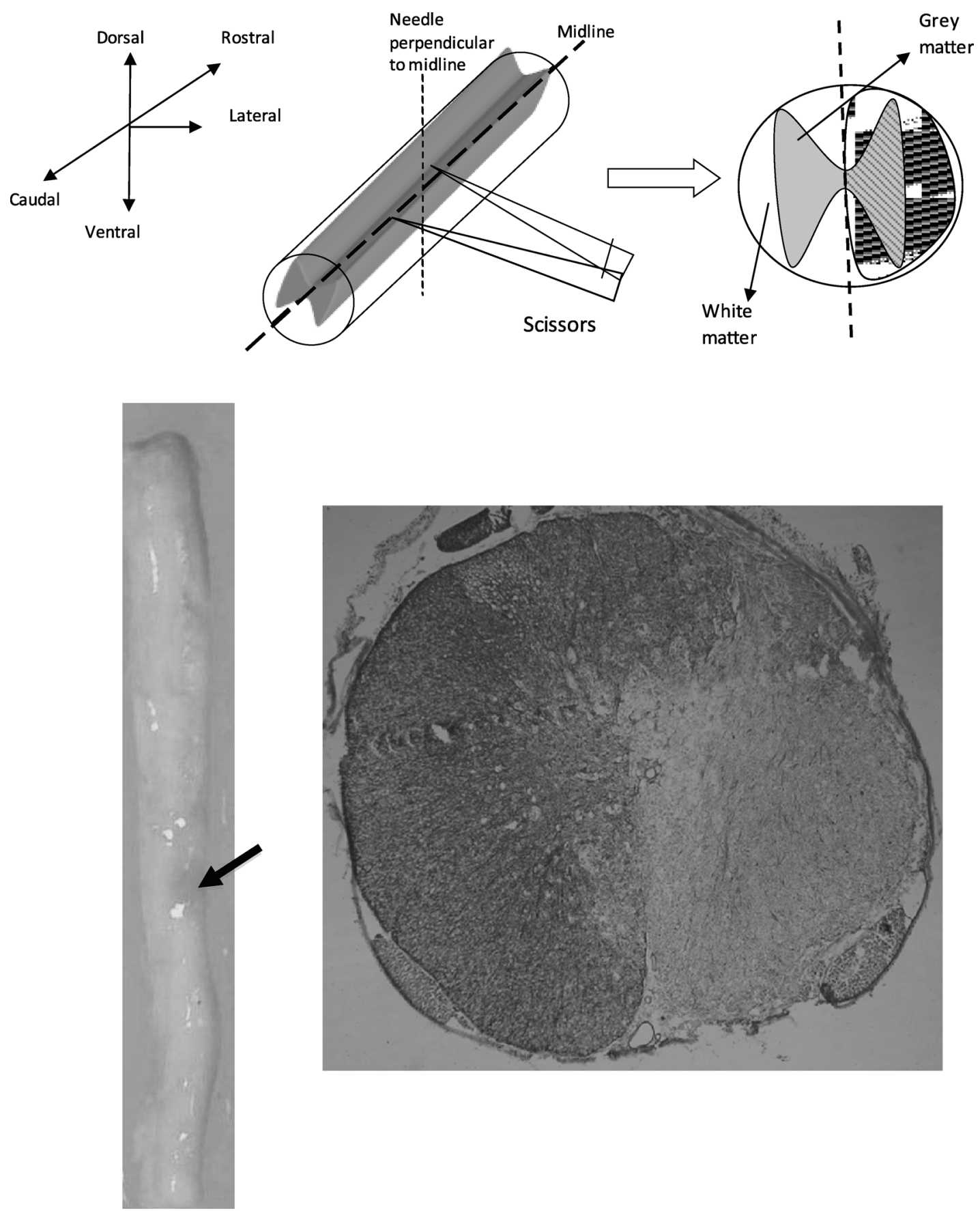

Fig. 1 A schematic of the hemisection surgery. Also shown is a transverse cross section of the hemisected spinal cord. The light micrograph shows an injured spinal cord. The arrow points toward the injured portion. A representative cross section of the injury site is also shown.

\subsection{Raman Spectroscopy}

A custom built Raman spectroscope was used for this study. ${ }^{12}$ The laser wavelength was $785 \mathrm{~nm}$ (Process Instruments, Salt Lake City, Utah) and spectra were collected in backscatter mode. The laser power at the sample was $450 \mathrm{~mW}$ and the spot size was $300 \mu \mathrm{m}$ determined using Zap-it ${ }^{\mathrm{TM}}$ paper. A fused silica cuvette was employed for in vitro experimentation on liquid samples. The dispersed light was detected using a Roper Scientific/PAR CCD camera and a Kaiser f/1.4 Holospec spectrograph. The spinal cord was oriented similar to its orientation in vivo with the dorsal side facing up. The cord was placed in a specially designed quartz holder that was placed on a 3-D stage controlled with micrometers and care was taken to keep the spinal cord hydrated with PBS during the course of an experiment. For hemisected cords, spectra were obtained focusing on the scar (12 spectra, 5 min each). For contused cords, as shown in Fig. 2, spectra were sequentially obtained beginning at an uninjured region at the rostral end of the cord and moving through the injury epicenter toward uninjured regions caudally ( $5 \mathrm{~min}$ per spectrum, spectral locations spaced $500 \mu \mathrm{m}$ apart). For healthy cords, spectra were obtained from random locations on the cord (at least ten spectra per cord, 5 min each). Further, spectra were 


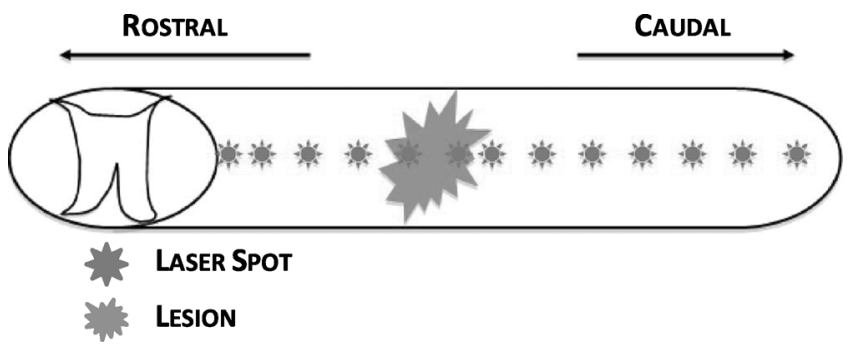

Fig. 2 Schematic of spectrum collection from a contused spinal cord. Spectra were collected moving from healthy uninjured portions at the rostral end through the glial scar, back to uninjured regions at the caudal end.

also obtained focusing on the injury epicenter (12 spectra, 5 min each). All experiments were done at room temperature.

\subsection{Data Processing and Analysis}

The separation of the fluorescence and Raman contributions to the observed Stokes shifted emission from near-infrared (NIR) excited ex vivo or in vivo tissue samples cannot be deterministically executed, unless it is actually known how much of each emission is present. As is often the case with biological samples, this information is not available. The primary goal in this study was to determine if healthy spinal cord can be discerned from injured spinal cord using Raman spectroscopy so an approach that can be potentially automated is utilized. Further, this approach is statistically unbiased in the sense that only deterministic, wellcharacterized, and reproducible artifacts are induced. ${ }^{15,16}$ This allows for comparison of spectra of different samples to each other visually and quantitatively, using statistical measures of differences such as integrated peak areas, in order to deduce whether changes occur due spinal cord injury.

Unless the fluorescence and Raman contributions can be separated by some independent method, all baseline correction procedures ${ }^{15,16}$ for biological samples contain assumptions and therefore induce distortions. For example, all procedures assume that the shapes of fluorescence spectra are featureless with respect to having relatively narrow peaks, e.g., choosing arbitrarily $\approx 200 \mathrm{~cm}^{-1}$ or less. To the extent that Raman features, including congested clumps of Raman features, are significantly less than $200 \mathrm{~cm}^{-1}$ wide, the smoothing procedure used produces a baseline that roughly bisects only the Raman contribution to the observed spectrum above the contribution formed by the sum of the fluorescence component and any Raman contribution that cannot be resolved due to spectral congestion. This is true because the wide smoothing window we use is 101 points wide corresponding to $\approx 200 \mathrm{~cm}^{-1}$. Thus in general, features that are much narrower than $200 \mathrm{~cm}^{-1}$ are reproduced in the baseline corrected spectrum without distortion. To the extent resolved Raman features are roughly $200 \mathrm{~cm}^{-1}$ wide and wider, the 101 point smoothed spectrum obtained from the raw data contains a contribution from those features and so their contribution will be decreased in, i.e., be subtracted from the raw data to obtain, the ultimate baseline corrected Raman spectrum. The baseline corrected spectrum thus contains only contributions from the resolved Raman spectrum without making any unjustified assumptions about the fluorescence spectrum or unresolved Raman components. However, the position of zero counts is completely unknown and the baseline corrected spectrum has positive and negative excursions from zero. One can properly correct for this when integrating peak areas by a proper choice of zero taken from the wings of the peak as described below.

Note that the cropping of the low Raman shift end of the spectrum precludes the net spectral shape of the fluorescence and the laser line rejection filter from making any contribution in shape or strength to the baseline corrected spectrum up to at least $\approx 1200 \mathrm{~cm}^{-1}$ Raman shift. Interestingly, relatively strong and wide features, e.g., amide I $\left(1670 \mathrm{~cm}^{-1}\right)$ and $\mathrm{CH}_{2}$ deformation $\left(1450 \mathrm{~cm}^{-1}\right)$, separated by a couple hundred $\mathrm{cm}^{-1}$, also make a contribution to the smoothed baseline and so these two features are also distorted, i.e., decreased by roughly $10 \%$ relative to all the other Raman features in the baseline corrected spectrum. The large clump that includes amide III $\left(\approx 1200-1300 \mathrm{~cm}^{-1}\right)$ is not affected as much but it certainly is systematically decreased.

Indeed we have previously shown ${ }^{17,36}$ that essentially the same "quantitative" results can be obtained (from spectra of phantoms, tissues in vivo, and simulations having very similar characteristics as those presented in this manuscript) with or without the same 101-7 smoothing baseline correction described below. For our spectral resolution, 101 points is sufficiently wide and in observing the spectra with the naked human eye, the relative heights of Raman features are compared with each other essentially as an implicit internal standard and it is these comparisons that have been highlighted, i.e., not "absolute" variations. Also, the seven point smoothing portion of this procedure (after spike removal) has no effect whatsoever.

Using Matlab ${ }^{\circledR}$ custom written routines, spectra were cropped in the fingerprint region $\left(400-1800 \mathrm{~cm}^{-1}\right)$ and were corrected for background fluorescence using an arbitrary but unbiased 101-point moving window-averaging scheme. ${ }^{12,17-19}$ This 101-point moving average was subtracted from the raw data and these background corrected spectra were smoothed using a seven-point moving window algorithm and where noted, spectra were standard normal variate (SNV) transformed by mean centering and dividing by the standard deviation. ${ }^{13,14}$ Fluorescence in the fingerprint region was calculated by integrating the raw counts to obtain the area under the curve and assuming the contribution of the Raman to the total emission is negligible. ${ }^{12,18,20}$ In order to make quantitative comparisons, seven distinct Raman peaks were indentified in the spectra and areas under each of the peaks were calculated from the baseline corrected, SNV transformed data. For quantitative analysis each of the peaks was assigned an individual baseline ${ }^{17}$ by subtracting the minimum intensity, i.e., from the wings of the peak in order to avoid negative values and subsequently summing the intensities across the wavenumbers defining the peak to obtain an area measure. The $\mathrm{SNV}^{13,14}$ transform was used to normalize for baseline shift and gain differences. Currently, we cannot discern that it produces any modification to the spectra or to the relative strength of the Raman features at all. The purpose of using the SNV transform is to allow averaging of spectra taken at different times from comparable samples with equal statistical weighting.

A one way analysis of variance (ANOVA) was performed followed by a Tukey's least significant difference (LSD) posthoc test to determine differences in peak areas between groups with $95 \%$ confidence. Further, as an independent measure of classifying the spectral peak areas, we implemented the k-nearest neighbor (KNN) algorithm in Matlab. 
Data were arranged as peak areas corresponding to the injury type (healthy, hemisected four days, two weeks or eight weeks PI, and contused cords). The training set was 50 randomly selected peak areas from the different injury conditions (healthy, hemisected, or contused) and the classification set was the remaining dataset. The algorithm based all classifications on a $k$-value of five nearest neighbors. This process was repeated 5000 times to obtain an average classification rate.

\subsection{Immunofluorescence and Histological Staining}

A total of 16 animals (four per group) were used for immunohistochemical and histological staining including healthy/uninjured controls, and four days, two weeks, and eight weeks after spinal hemisection injury. Animals were euthanized with an intraperitonial injection of sodium pentobarbital $(50 \mathrm{mg} / \mathrm{kg})$, followed by transcardial perfusion with PBS $(500 \mathrm{ml})$ prior to perfusion with $4 \%$ paraformaldehyde in PBS $(500 \mathrm{ml})$. Excised spinal cords were left overnight in $4 \%$ paraformaldehyde and then placed in 20\% Sucrose solution in PBS for two days. Next an 8 $10 \mathrm{~mm}$ segment of each spinal cord including the injured region, were frozen in optimal cutting temperature media and $20-\mu \mathrm{m}$ serial sections were cut in the transverse plane, using a cryostat. Series of serial sections separated by $100 \mu \mathrm{m}$ were mounted on glass slides. One series was immune-stained for CSPGs (CS-56, mouse monoclonal antibody for intact CSPGs, 1:100, Sigma C8035). This series was first incubated in blocking media (10\% normal goat serum, $3 \%$ bovine serum albumin in PBS, pH 7.4) overnight. Next, sections were incubated with a secondary antibody [AlexaFluor ${ }^{\circledR} 555$ (red) IgM goat anti-mouse, Invitrogen Inc.] for one hour at room temperature and stored at $4{ }^{\circ} \mathrm{C}$ until imaged. A histological stain for myelin using the eriochrome cyanine staining technique was also employed on a series of sections. Both fluorescent and brightfield imaging was done using a Zeiss Axioskop microscope (Carl Zeiss, Oberkochen, Germany). Digital images were captured using a Spot RT Slider camera (Diagnostic Instruments, Sterling Heights, MI) and the accompanying Spot Advanced software (v.3.3.4 for Macintosh, Diagnostic Instruments).

\section{Results}

\subsection{Raw Data of Healthy and Injured "Hemisected" Spinal Cords and Variation in Fluorescence}

Figure 3(a) shows the unprocessed data obtained from various locations on a single healthy spinal cord and Fig. 3(b) shows the representative time course on a single location of a particular cord for successive 5-min CCD acquisitions, respectively. The integrated fluorescence values are shown on the ordinate. It can be seen that there is background fluorescence but the Raman peaks are evident and that the relative magnitude of these peaks is similar (for each injury condition), irrespective of the fluorescence, although eight of ten locations on the healthy cord produced data within $\pm 3 \%$ of the average. The other two locations produced a large bivariate deviation from average and the variation is likely due to uneven drying of the tissues, the presence of surface wrinkles or creases, or the penetration of the
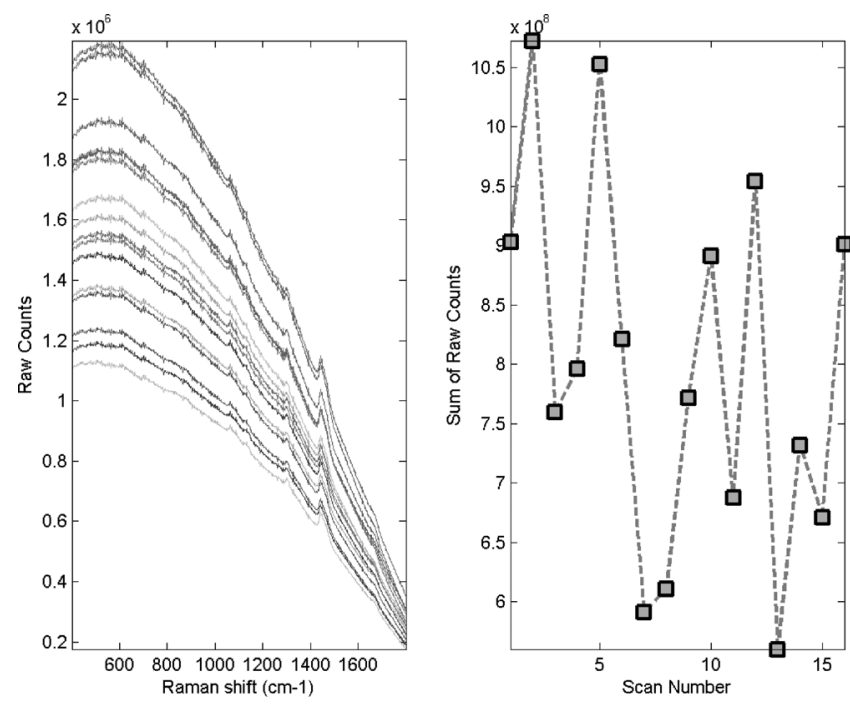

(a)
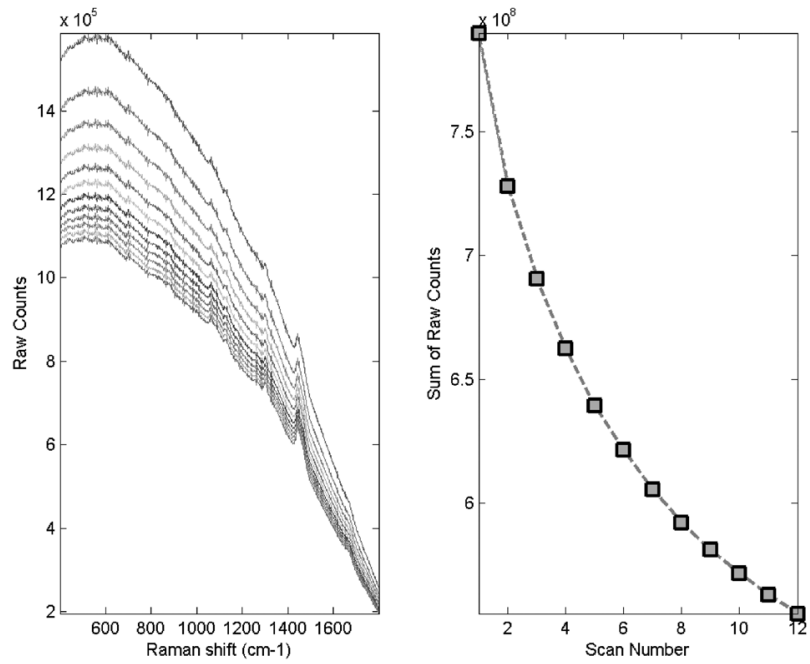

(b)

Fig. 3 (a) Raw data from various locations on a single spinal cord. Also shown on the right are integrated fluorescent counts for the raw spectra. Note that although background fluorescence varies between locations, the Raman features are fairly constant. Exposure time per location was $5 \mathrm{~min}$. (b) Raw data from extended irradiation of a single point on a spinal cord. Integrated fluorescence is shown on the right and photobleaching of the fluorescence is apparent. Note that the Raman features are similar irrespective of the fluorescence. Exposure time per spectrum was $5 \mathrm{~min}$.

excitation laser through the tissues to the quartz holder or some combination of one or more of these factors.

For most of the data, the fluorescence variation with time is commensurate with the variation observed by sampling spectra from different locations on the same cord. Further, it is also clear that the spectra contain Raman features that are relatively constant with respect to the fluorescence, indicating that the location did not affect the relative Raman spectral features. In order to see this more clearly, Fig. 4(a) shows the average and the $95 \%$ confidence intervals of all of the raw spectra taken either on healthy tissue or different locations within the SCI zone for each injured cord at each time. The fluorescence for the healthy cord was substantially larger $(p<0.005)$ and spectrally 

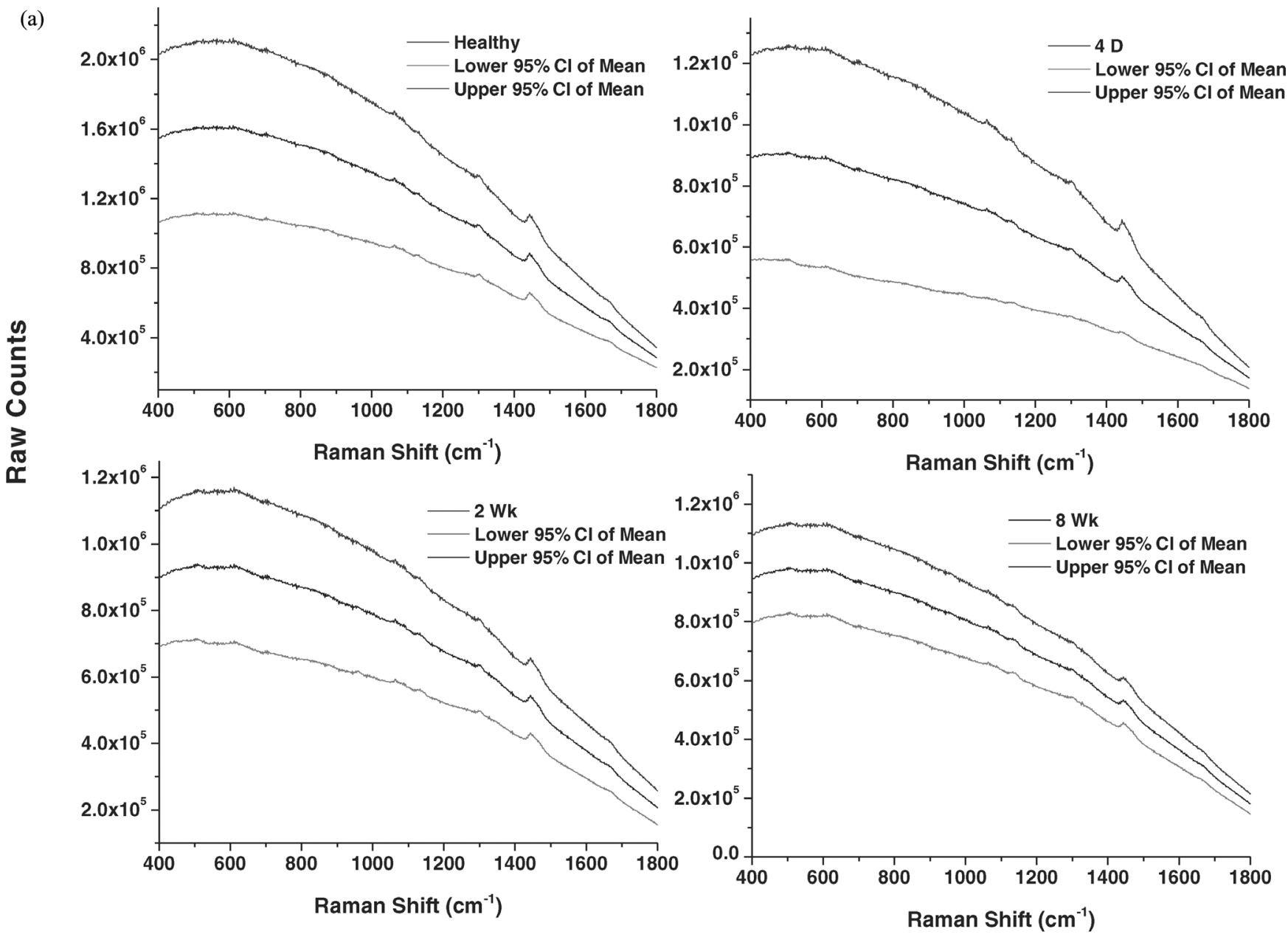

(b)

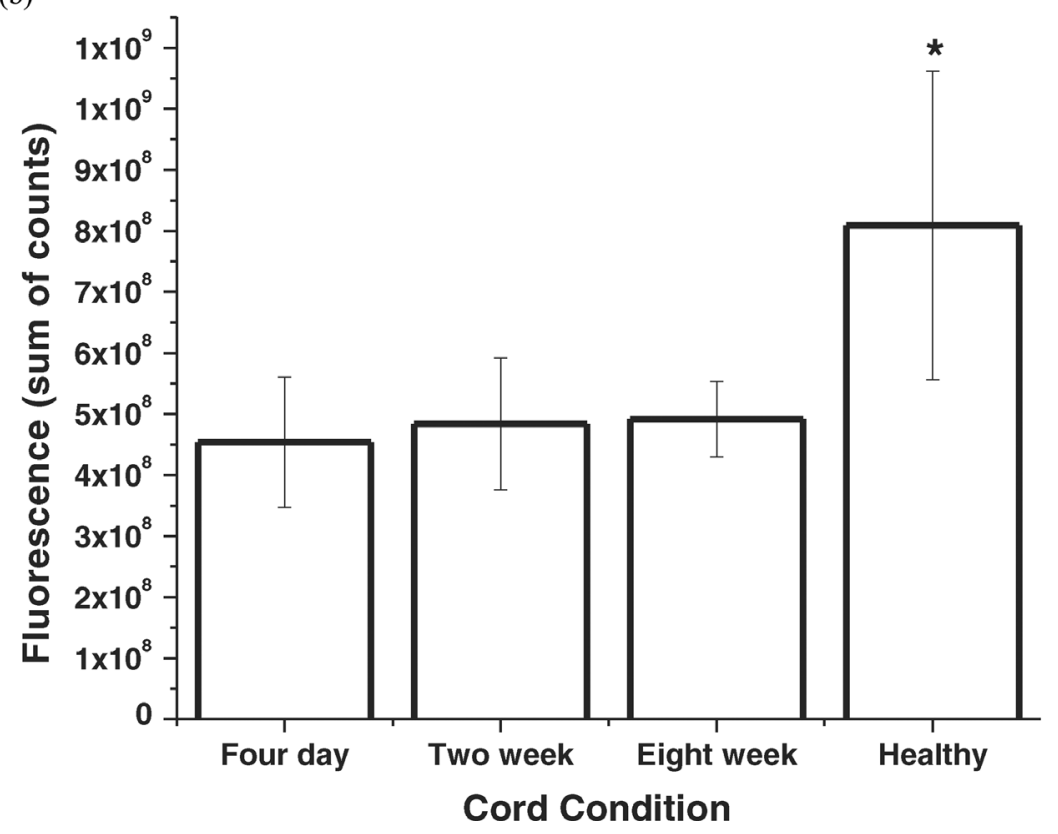

Fig. 4 (a) Raw data for the hemisected and healthy spinal cords. Data are plotted as the mean raw data obtained from all animals per group and the upper and lower $95 \%$ confidence interval. Note the different scales on the ordinate. (b) Fluorescence measures for each group after hemisection injury. Data are plotted as mean \pm standard deviation. Asterisk $\left(^{*}\right)$ indicates that the healthy cords had significantly higher fluorescence $(p<0.005)$ than injured cords. No significant differences in fluorescence between injured cords were noted. 


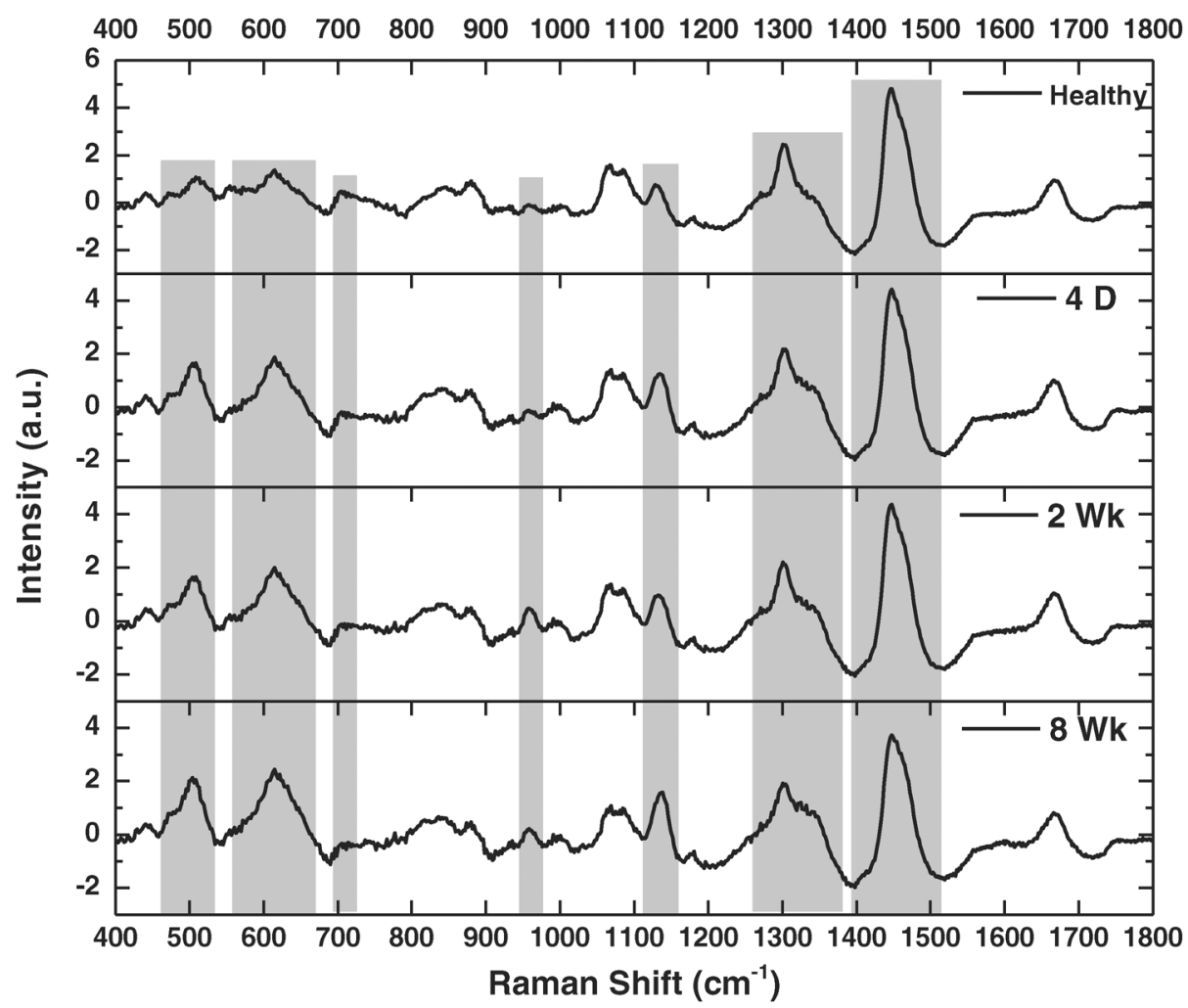

Fig. 5 The mean background corrected, SNV-transformed spectra of hemisected and healthy spinal cords. Important bands are highlighted in gray. Please refer to Tables 1 and 3 for peak assignments.

narrower than for any of the injured rats. The fluorescence of the injured cords monotonically decreased with time post-injury but no significant difference in fluorescence between injured cords was observed. This is shown in Fig. 4(b).

\subsection{Raman Spectra of Healthy and Hemisected Spinal Cord at Different Times Post-injury}

Figure 5 shows the mean baseline-corrected, smoothed, and transformed spectra of control and hemisected spinal cords. Although these data are averages over many animals, the variability in the data was small and very systematic variation could be perceived. Certain features such as the $1450 \mathrm{~cm}^{-1} \mathrm{CH}_{2}$ deformation mode and the $1670 \mathrm{~cm}^{-1}$ amide I modes were very prominent in all the spectra. The peak at $700 \mathrm{~cm}^{-1}$, corresponding to cholesterol, decreases at four days PI and is absent at two and eight weeks PI, in comparison with the healthy cords. Important peaks are highlighted and peak assignments are listed in Table 1 . In order to clearly see the variation, we produced difference spectra using the healthy cord as reference (Fig. 6), which revealed very systematic variations that were often, but not always, monotonic. We observed in Fig. 6 that relative to the healthy spinal cord tissue, the tissue in and around injured spinal cord showed a decrease in the peak intensity at $700 \mathrm{~cm}^{-1}$ (cholesterol), and monotonic decreases in the peaks at $1005 \mathrm{~cm}^{-1}$ (white matter, phenylalanine breathing mode in proteins), 1065 and 1086 $\mathrm{cm}^{-1}\left(\mathrm{C}-\mathrm{C}\right.$ stretching and $\mathrm{PO}_{2}$ stretching of lipids and phospholipids), $1299 \mathrm{~cm}^{-1}$ ( $\mathrm{CH}_{2}$ twisting and wagging), and 1450 $\mathrm{cm}^{-1}\left(\mathrm{CH}_{2}\right.$ deformation from lipids and proteins) features and a monotonic increase in the peak intensities at 490 and $640 \mathrm{~cm}^{-1}$ (CSPGs and GAG aggregation). This indicates that the tissue damage and the subsequent wound healing response post-SCI, led to a change in the concentration of the biomolecules that contributed to these spectral peaks. Other features, notably near $800-1000 \mathrm{~cm}^{-1}$ and above $1500 \mathrm{~cm}^{-1}$, had quite complex behavior with respect to time post-injury. Uninjured rats were used as opposed to sham-operated rats as controls. This was done in order to compare uninjured cords from healthy animals to cords from injured animals. Also, the injury model used was not a novel model of SCI. It was further observed, that spectra from uninjured portions of healthy cords showed features similar to those from control animals (data not shown), thereby precluding the use of sham animals.

\subsection{Immunofluorescence and Histology of Hemisected Spinal Cords}

Figure 7 shows the results from the immunofluorescent staining of spinal cord sections for CSPGs (top panel) and the histological staining of sections for myelin (bottom panel). The immunostaining for CSPGs, indicates that the intensity of labeling is maximal at two weeks PI, but is above background levels at four days and eight weeks PI relative to uninjured controls. Myelin staining indicate that at four days PI, demyelination is present but is incomplete on the side of injury. By two weeks PI, there is intense demyelination on the ipsilateral side of injury, which continues at eight weeks PI. The Raman spectra complement the 
Table 1 Peak assignments to Raman spectra of spinal cord tissue. Question marks indicate unassigned peaks.

\begin{tabular}{|c|c|}
\hline Wavenumber $\left(\mathrm{cm}^{-1}\right)$ & Peak Assignment \\
\hline 510 & CSPG aggregation \\
\hline 617 & GAGs \\
\hline 701 & Cholesterol \\
\hline 794 & $?$ \\
\hline 813 & C-OS linkage \\
\hline 840 & $?$ \\
\hline 880 & $?$ \\
\hline 958,997 & C-O-C, C-O-S \\
\hline 1069,1085 & $\mathrm{SO}_{3}{ }^{-}$, Cholesterol C-C stretch \\
\hline 1137 & $\mathrm{C}-\mathrm{H}, \mathrm{C}-\mathrm{OH}$ of glucose derivatives \\
\hline 1177 & $?$ \\
\hline 1304 & $\mathrm{CH}_{2}$ twist \\
\hline 1448 & $\mathrm{CH}_{2}$ Bending \\
\hline 1567 & Heme \\
\hline 1660 & Amide I \\
\hline 1750 & $\mathrm{COOR} / \mathrm{COOH}$ \\
\hline
\end{tabular}

results of the histological and immunohistochemical assessment of the post-injury lesion site.

\subsection{Raw Data of Healthy and Injured "Contused" Spinal Cords and Variation in Fluorescence}

Spectra from contused cords showed reduced fluorescence in comparison to healthy cords, similar to the spectra obtained for hemisected cords. Upon scanning across the spinal cord from uninjured portions, through the injured glial scar locations, back to healthy uninjured regions, the fluorescence decreased in the injured regions and increased back to normal levels with greater distance from the lesion site (data not shown).

\subsection{Raman Spectra of Contusion Injured Spinal Cords}

Figure 8(a) shows the mean baseline-corrected, smoothed, and transformed spectra of data obtained from the contusion epicenter. In order to see the variation with respect to uninjured controls, we produced difference spectra using the healthy cord as reference [Fig. 8(b)], which revealed difference spectra very similar to hemisected cords. Figure 8(c) shows the Raman spectra from scanning across a contused cord from healthy to injured to healthy regions. Important peaks are highlighted and peak assignments are listed in Table 1. It can be observed that spectral features considerably change as one approaches the injury site and return to the appearance of uninjured controls as one moves to uninjured regions. Figures 8(d) and 8(e) show the changes in peak areas corresponding to spectra in Fig. 8(c). The peak areas corresponding to GAGs and lipids are shown and it was noted that at the injury site, the peak areas for GAGs increase whereas those corresponding to lipids decrease.

\subsection{Raman Spectra of Chondroitin Sulfate Glycosaminoglycans}

Figure 9 shows the room temperature time evolution of the PBS subtracted, baseline-corrected Raman spectrum of chondroitin-6 sulfate in PBS (pH 7.4), treated with cABC (2 Units/ml). Chondroitin sulfate consists of a long polysaccharide chain with free carboxylic acid, sulfate, and methyl amide groups as well. The spectrum of the undigested material displays well-known features at $600 \mathrm{~cm}^{-1}$ and below, corresponding to ring vibrations of the individual saccharides, and at $1064 \mathrm{~cm}^{-1}$, corresponding to the sulfate group. In addition, we observe overlapping Raman features near the anomeric carbon linkage mode near 800-900 $\mathrm{cm}^{-1}$, as well as a variety of features about $1600 \mathrm{~cm}^{-1}$ corresponding to the amide linkages and carboxylic acid carbonyl localized modes. ${ }^{10,21,23}$

\subsection{Peak Area Quantification and Classification using k-Nearest Neighbor Algorithm}

Peak areas were calculated as described in Sec. 2 and the results of the one-way ANOVA, followed by a Tukey's LSD post-hoc test are shown in Table 2. The groups having the same letters are not significantly different. As shown in Table 2, peak 3 (1210 $\left.1396 \mathrm{~cm}^{-1}\right)$ and peak $5\left(1021-1113 \mathrm{~cm}^{-1}\right)$ showed no significant differences. However, many significant differences between groups were observed for the remaining peaks. Peaks corresponding to healthy cords were significantly different than injured spinal cords although fewer significant differences within injury groups were noted. The KNN algorithm was used as an independent measure to classify the spectra and the resultant classification matrix from the KNN algorithm is shown in Table 3. The overall true classification rate observed was $83 \%$. The classification matrix indicates the percentage of correctly classified data sets. For example, reading across the second row of Table 2, 97\% of the healthy cords were correctly classified as healthy and the remaining $3 \%$ were incorrectly classified as hemisected cords. The four-day hemisected cords had a low classification rate of $48 \%$, whereas the two-week hemisected and contused cords had classification rates of 94 and $96 \%$, respectively. The eight-week hemisected cords had a classification rate of $63 \%$ but importantly, the percentage of injured cords that got classified as uninjured was extremely low for the two- and eight-week cords, with the four-day cords having a false classification of $12 \%$.

\section{Discussion}

There are clearly some systematic changes in the spectra that bear discussion in the context of the known development of the glial scar that was reviewed earlier and the known strengths and limitations of spontaneous Raman spectroscopy. The results also suggest some improvements in experimental 


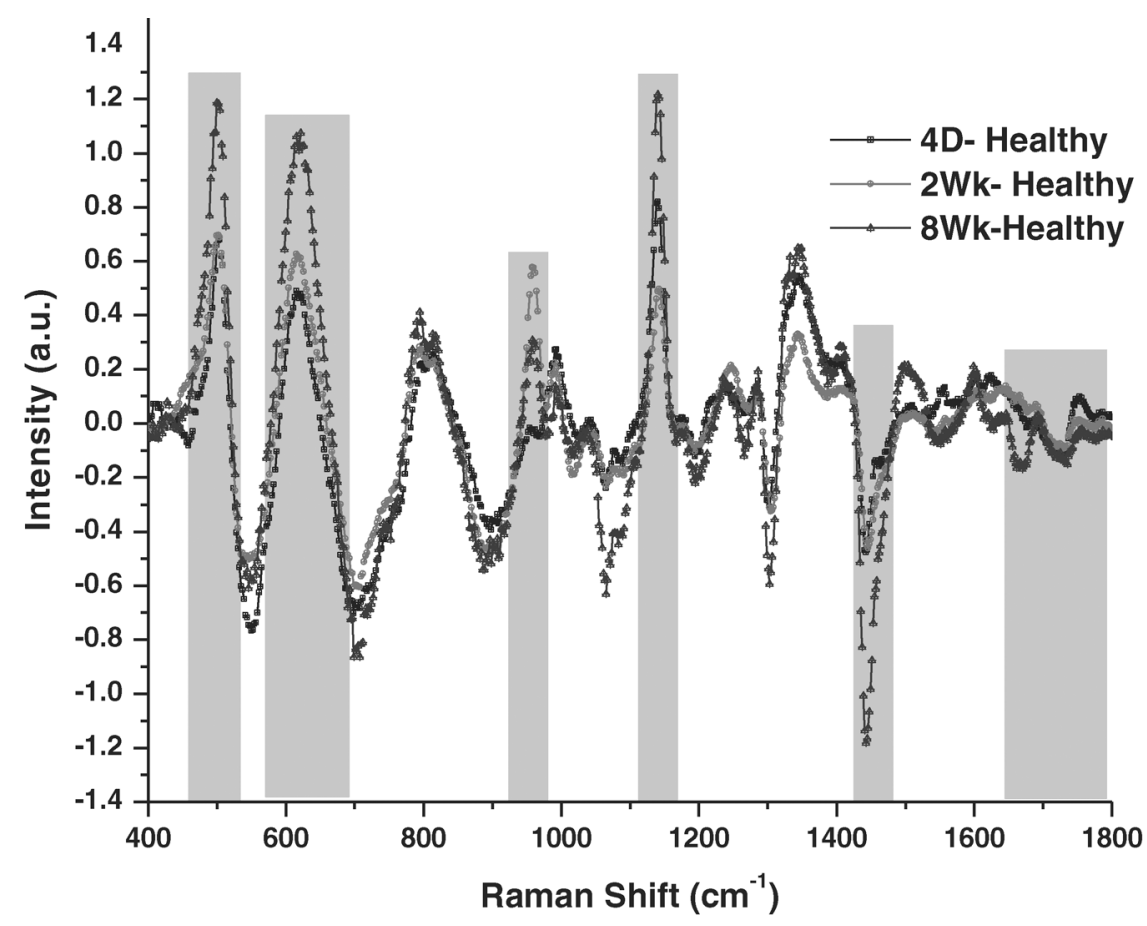

Fig. 6 Difference spectra of the hemisected cords using healthy cords as a reference. Important peaks are highlighted with gray bands. Note that there appear to be systematic changes that occur with progression of injury. Important changes corresponding to GAGs and proteoglycans (450$540 \mathrm{~cm}^{-1}, 541-690 \mathrm{~cm}^{-1}$, and 940-995 $\mathrm{cm}^{-1}$ ), demyelination (lipid loss, 1398-1519 $\mathrm{cm}^{-1}$ ), and carbonyl chemistry (above $\left.1650 \mathrm{~cm}-1\right)$ are highlighted. Please refer to Tables 1 and 3 for peak assignments.
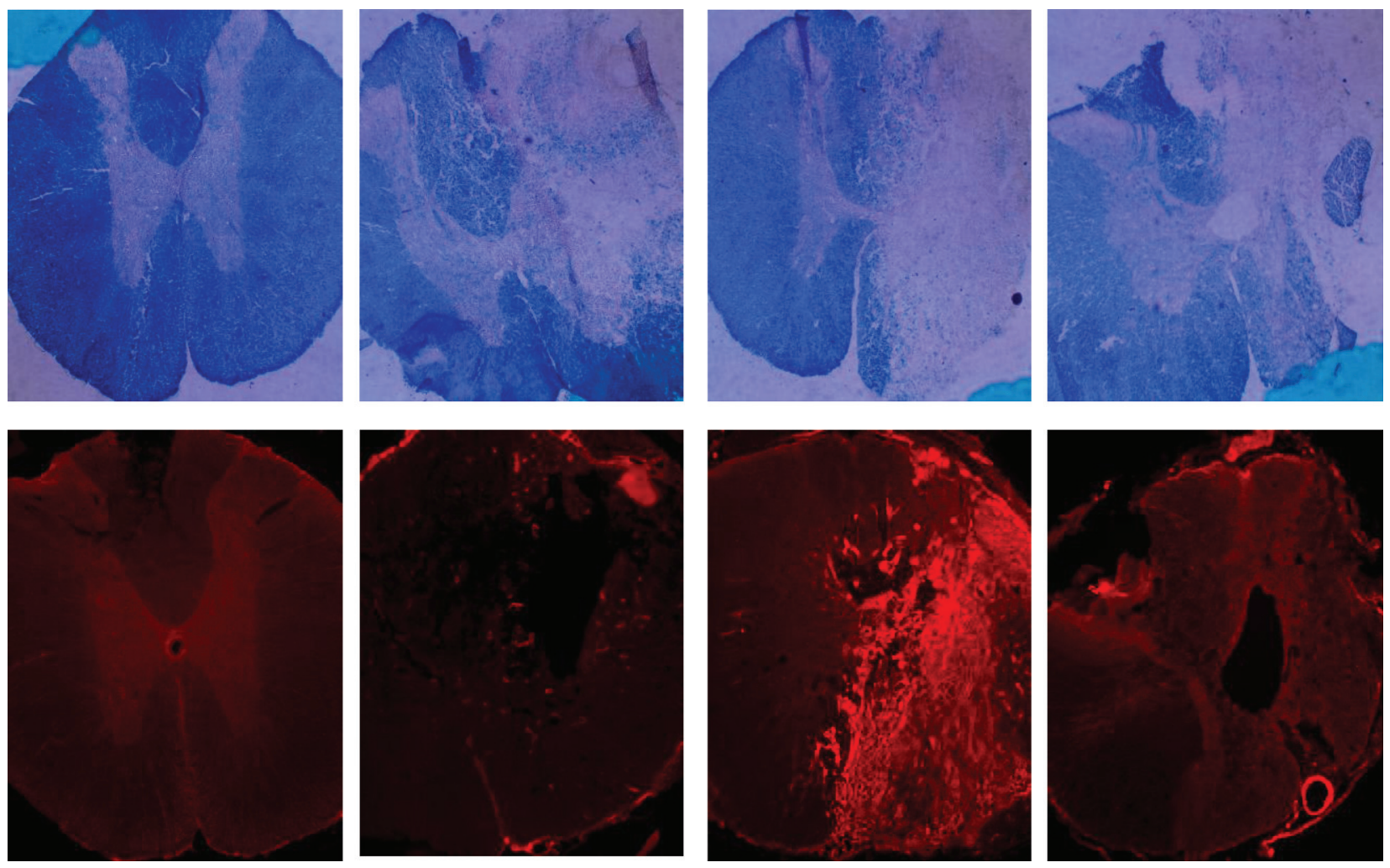

Fig. 7 Representative Immunohistochemical photomicrographs of healthy and hemisected spinal cords. Photomicrographs are temporally arranged from left to right as healthy, four days, two weeks, and eight weeks PI. Top row: Eriochrome cyanine stain for myelin. Healthy spinal cord stains blue and the gray and white matter are distinctly different. At four days $\mathrm{Pl}$, demyelination is evident but not complete, at two weeks $\mathrm{PI}$, there is complete demyelination on the ipsilateral side of injury which continues at eight weeks PI. Bottom row: CS-56 immunofluorescent stain for CSPGs. The healthy cords have a uniform background staining intensity. At four days PI, there is a slight increase in intensity of staining. At two weeks PI, the intensity of staining is clearly maximal on the ipsilateral side and at eight weeks $\mathrm{PI}$, there is increased intensity in comparison with controls, but not as intense as the two weeks PI staining. 

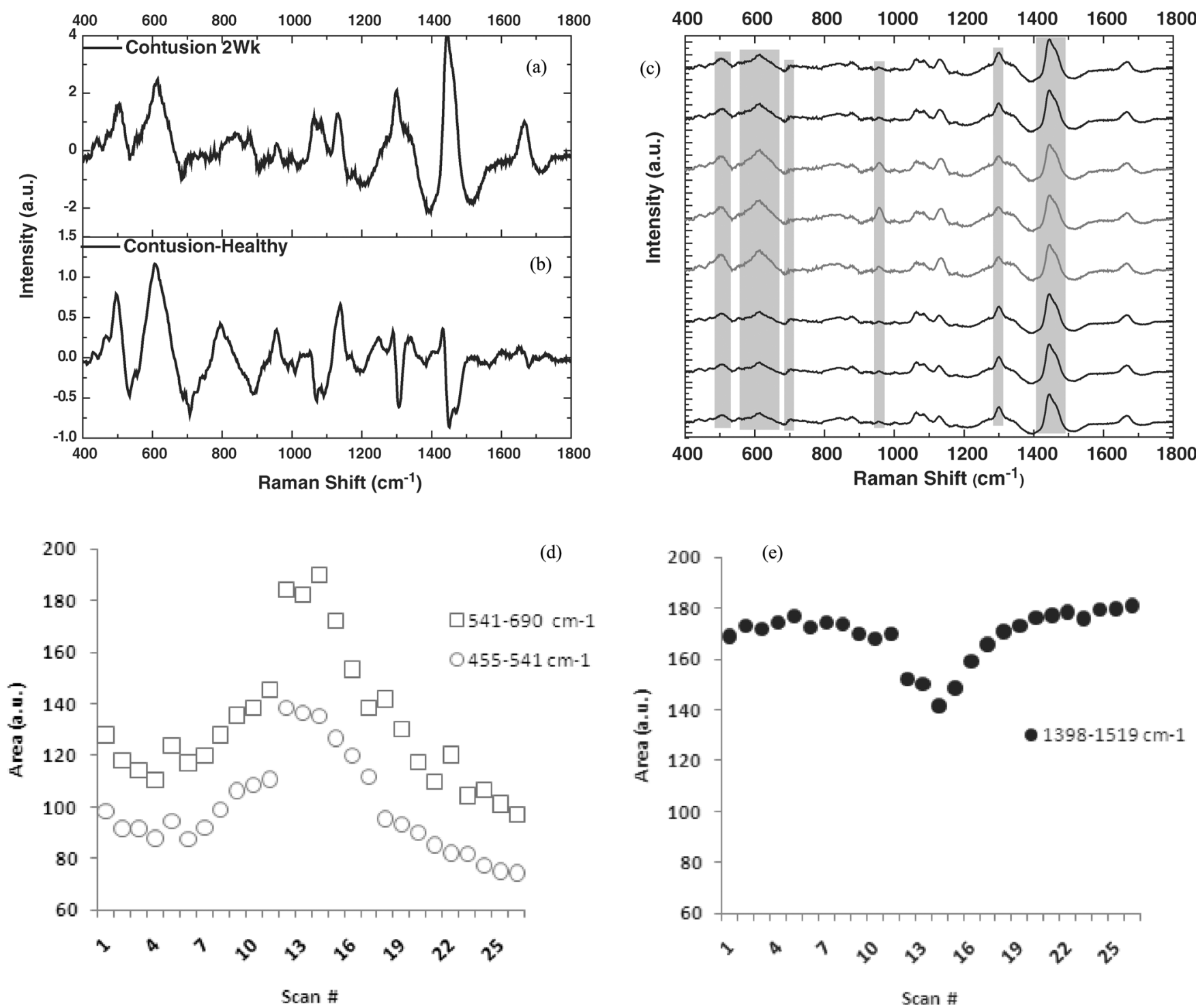

Fig. 8 (a) Mean SNV transformed spectrum of contused spinal cords. (b) Difference spectrum of contused cords using healthy cords as a reference. (c) Raman spectra from a scan across a contused spinal cord. Rostral is top and caudal is bottom. Spectra in black are from healthy uninjured regions and those in red are from the scar site. Important bands are highlighted in gray. Note the change in spectral features around the 400-800, 940-1000, and 1400-1500 $\mathrm{cm}^{-1}$ regions. Please refer to Tables 1 and 3 for peak assignments. Peak areas corresponding to (d) aggregated CSPGs and GAGs and (e) lipids from a scan across a contused spinal cord. Scan numbers 1-8 and 17-27 are on uninjured locations and scan numbers 9-16 are on the scar site. The distance between each scan location was $500 \mu \mathrm{m}$. For GAGs and CSPGs, the peak area increases upon approaching the scar and for lipids, decreases.

technique going forward and this will be discussed as well. The hallmarks of SCI are cell death, demyelination, and aggregation of GAGs and CSPGs. Peak assignments were made based exclusively on the literature pertaining to Raman spectroscopy of the central nervous system (brain tissue), ${ }^{22}$ GAGs, ${ }^{10,23}$ CSPGs, ${ }^{23,24}$ cholesterol, ${ }^{22,25}$ and blood. ${ }^{26}$ These are listed in Table 1. There are two types of Raman features that monotonically change post-injury. The decrease in the $700 \mathrm{~cm}^{-1}$ cholesterol peak ${ }^{21}$ and $1450 \mathrm{~cm}^{-1} \mathrm{CH}_{2}$ deformation Raman activity, corresponding to demyelination of the axons and cell death; and the large increase in Raman intensity below 600 $\mathrm{cm}^{-1}$, which is associated with increased CSPG expression and GAG aggregation. ${ }^{23}$ These results are also well supported by the results of the immunofluorescent and histological characterization of injured and healthy spinal cord tissue reported in this study, which indicated increased CSPG levels and severe demyelination post-injury. There was also an increase in the peak area in the region of $950-1000 \mathrm{~cm}^{-1}$, corresponding to the vibrations of the glycosidic $\mathrm{C}-\mathrm{O}-\mathrm{C}$ linkages and $\mathrm{C}-\mathrm{O}-\mathrm{S}$ linkages of GAGs. ${ }^{10,21}$ From the difference spectra in Fig. 6 it is observed that at two weeks PI, the peak area for this peak is maximum. This corresponds to a high expression of CSPGs at two weeks PI, as seen in the immunofluorescence results. This result corresponds well with findings by Jones et al. that showed that CSPGs have maximal expression at two weeks post-injury ${ }^{27}$ and persist at elevated levels for several weeks PI. $^{3,28}$ Also, upregulation of hyaluronic acid (HA) could contribute to the spectral changes related to CSPGs and GAGs. It is known that HA, which is a GAG, accumulates in injured spinal cord as gliosis proceeds, and high-molecular HA becomes 


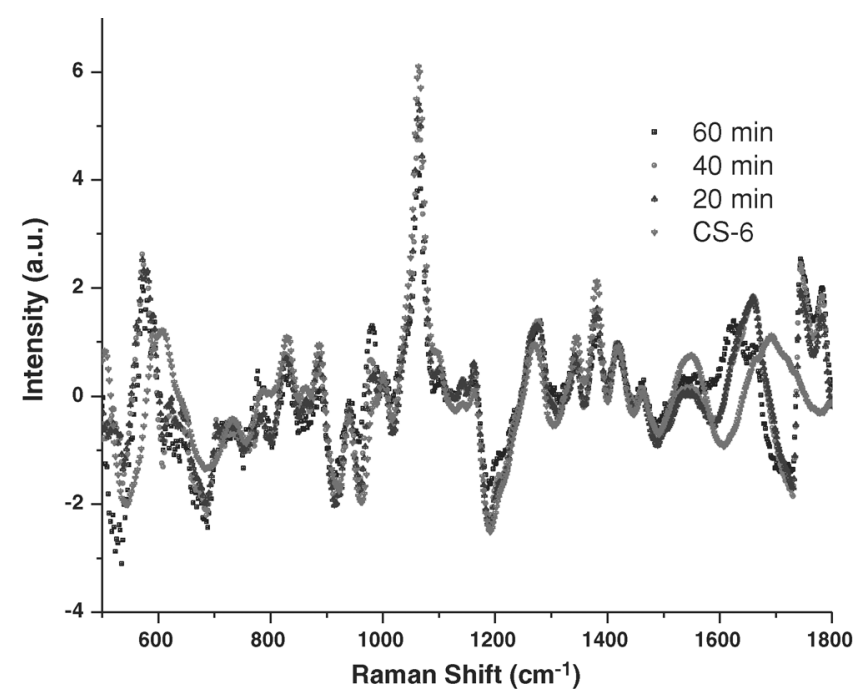

Fig. 9 Time course of $\mathrm{CABC}$ digestion of chondroitin 6-sulfate in PBS at room temperature. Spectra were obtained at intervals of $20 \mathrm{~min}$ for up to an hour. The spectrum of the CS- 6 solution is also shown. Note the evolution of the peaks around $1750 \mathrm{~cm}^{-1}$ upon digestion with cABC.

overabundant in the ECM surrounding glial scars with injury progression. ${ }^{29} \mathrm{HA}$ is also known to accumulate in demyelinated lesions. $^{30}$

But perhaps more interesting is the activity about $1600 \mathrm{~cm}^{-1}$, in which a range of new features appear, extending to rather high Raman shifts, both in the difference Raman spectra, as well as in the digestion spectra of CS-6 by cABC. It is well known that a variety of compounds, all containing the carbonyl group, display spectral features beyond $1670 \mathrm{~cm}^{-1} \cdot{ }^{10}$ Due to the generally weak character of spontaneous Raman scattering, easily observable changes must correspond to materials that are in high concentration in the probed volume. Due to the presence of the carbonyl related peaks in the difference spectra (Fig. 6) and the in vitro digestion of CS-6 (Fig. 9), we hypothesize that the conditions in the injured cord are conducive to acid catalyzed hydrolysis of the polysaccharide chains present on the CSPGs and GAGs in the probed volume of injured tissue. This would be expected to be a slower process in vivo, but in essence, with the same reaction products as the $\mathrm{cABC}$ digestion. Due to the inflammatory response, the local medium at a wound site is known to be increasingly acidic. ${ }^{31,32}$ Acid catalyzed reactions are expected to be slower in vivo, and we note that the observed changes take place over weeks in injured cord, but in less than an hour in the $\mathrm{cABC}$ catalyzed reaction. In the case of injured spinal cord tissue in vivo, monosaccharides and multisaccharides are directly produced; but in the $\mathrm{cABC}$ digestion only disaccharides are first produced, which we hypothesize are then hydrolyzed in situ to produce monosaccharides. Taking this one step further, we propose that the monosaccharides, being in equilibrium with their linear chain forms, produce lactones as more monosaccharides are produced. Because the lactones have a rather high carbonyl Raman shift, about $1750 \mathrm{~cm}^{-1}$, they are more easily observed than some other species due to a lack of spectral congestion. ${ }^{10}$ It is also known that lipid peroxidation takes place post-SCI and produces compounds containing carbonyl groups. ${ }^{32}$ The contribution of these compounds cannot be entirely discounted.

In order to make quantitative measurements, we calculated peak areas of seven spectral regions that are listed in Table 2. The peak corresponding to the amide III region (peak 3, 1210-1396 $\mathrm{cm}^{-1}$ ) and peak $5\left(1021-1113 \mathrm{~cm}^{-1}\right)$, which is a spectrally complex peak, implying that many different functional groups are involved in the evolution of the peak, did not show significant differences between groups. However, the remaining five peaks

Table 2 Peak widths for quantitative calculations and results of post-hoc testing. Groups with the same letters are not significantly different ( $p>0.05)$.

\begin{tabular}{|c|c|c|c|c|c|c|c|}
\hline Peak \# & $\begin{array}{l}\text { Peak Width } \\
\left(\mathrm{cm}^{-1}\right)\end{array}$ & $\begin{array}{c}\text { Peak } \\
\text { Assignment }\end{array}$ & $\mathrm{p}$-Value & Healthy & 4 Day & 2 Week & 8 week \\
\hline 1 & $\begin{array}{c}1623- \\
1720\end{array}$ & $\begin{array}{l}\text { Amide I, } \\
\text { lactone, } \\
\text { carbonyl }\end{array}$ & 0.0293 & B & $A$ & $A$ & $A, B$ \\
\hline 2 & $\begin{array}{c}1398- \\
1519\end{array}$ & Lipids & 0.0035 & $A$ & B & B & B \\
\hline 3 & $\begin{array}{l}1210- \\
1396\end{array}$ & Amide III & 0.9245 & A & $A$ & $A$ & $A$ \\
\hline 4 & $\begin{array}{l}1114- \\
1167\end{array}$ & $\begin{array}{l}\text { GAGs, } \\
\text { CSPGs }\end{array}$ & 0.0002 & C & $A, B$ & $B, C$ & $A$ \\
\hline 5 & $\begin{array}{l}1021- \\
1113\end{array}$ & Composite & 0.3622 & $A$ & $A$ & $A$ & $A$ \\
\hline 6 & $541-690$ & $\begin{array}{l}\text { GAGs, } \\
\text { CSPGs }\end{array}$ & $<0.0001$ & C & B & B & $A$ \\
\hline 7 & $456-541$ & $\begin{array}{l}\text { GAGs, } \\
\text { CSPGs }\end{array}$ & 0.0088 & B & $A$ & B & $A$ \\
\hline
\end{tabular}


Table 3 Classification matrix output from KNN algorithm. The numbers indicate percentage of correct classification.

\begin{tabular}{|c|c|c|c|c|c|}
\hline & Healthy (1) & 4 day (2) & $2 \mathrm{Wk}(3)$ & $8 \mathrm{Wk}(4)$ & Contused (5) \\
\hline Healthy (1) & 97.4 & 0.002 & 1.73 & 0.83 & 0 \\
\hline 4 day (2) & 11.7 & 47.86 & 20.97 & 11.84 & 7.60 \\
\hline $2 \mathrm{Wk}(3)$ & 0.74 & 2.62 & 93.98 & 2.64 & 0 \\
\hline $8 \mathrm{Wk}(4)$ & 0.002 & 12.41 & 13.74 & 63.01 & 10.82 \\
\hline Contused (5) & 0.77 & 0.046 & 0.081 & 3.24 & 95.86 \\
\hline
\end{tabular}

showed significant differences and these peaks corresponded to GAGs (541-690 $\mathrm{cm}^{-1}$ and $456-540 \mathrm{~cm}^{-1}$ ) and lipids (1398-1519 $\left.\mathrm{cm}^{-1}\right)$, indicating changes in the concentrations of GAGs, and demyelination and cell death. No clear trend was noted in the differences between groups, with respect to time post-injury. However, it was clear that the healthy cords were different from the injured cords, as was also qualitatively evidenced from the Raman spectra. The results of the KNN classification algorithm demonstrated that injured cords could be differentiated from uninjured cords based on their spectral features. The spinal cords belonging to the four day post-injury group had the lowest classification rate. This may be due to the fact that during the acute stages of SCI, the wound site is highly dynamic and many of the processes that come into play in chronic stages have not yet begun. There were also substantial amounts of extravasated blood around the injury site that could not be perfused away. Further, this tissue was also very difficult to handle ex vivo due to its fragility. All of these factors lead to a lot of experimental variability as can be noted in Fig. 4(a) from the large spread in the distribution of the fluorescence and intensity of Raman peaks. The classification rates for the remaining groups were high with injured cords seldom being classified as uninjured and vice versa.

Since the contusion injury is less focal than a hemisection, ${ }^{4}$ we scanned across a contused cord and observed that upon approaching or leaving the injury site, the spectra contain Raman features that are both qualitatively and quantitatively distinct from the uninjured sites. The peak areas corresponding to aggregated proteoglycans and GAGs (541-690 $\mathrm{cm}^{-1}$ and $\left.456-540 \mathrm{~cm}^{-1}\right)^{10,21,23}$ showed a trend of increasing in and around the injury site. This is consistent with the observation that the CSPGs, which have maximal expression at two weeks post-injury, ${ }^{27}$ surround the lesion core and decrease in concentration away from the injury site. Corroborating the upregulation of CSPGs is the observation that there was an increase in the peak area in the region of 950-1000 $\mathrm{cm}^{-1}$, corresponding to the vibrations of the glycosidic C-O-C linkages and C-O-S linkages of GAGs. ${ }^{10,21}$ The contusion injury is more common in the clinical scenario, ${ }^{33,34}$ thus this result is very promising in terms of developing Raman as a diagnostic or noninvasive monitoring tool for SCI since the spectra are essentially providing a spectroscopic representation of the injured spinal cord. We wanted to demonstrate that RS could be used to distinguish uninjured locations from injured portions of a spinal cord in a clinically relevant model of SCI. It was not our intention to use RS to distinguish between the two types of injuries (contusion and hemisection) reported here. Further, the wound healing responses to these injuries are very different. However, changes such as demyelination and CSPG upregulation are common to both injuries.

SNV-transformed data was used to compare Raman spectra of rat spinal cord tissues. First, it was noted that the observed fluorescence varied by as much as $15 \%$ depending on the exact location of probing, even in healthy tissue. Since $80 \%$ of the data from healthy cords is internally consistent to $\pm 3 \%$, and fluorescence cannot be produced unless the incident light penetrates the surface, we suggest that much of the observed variation in absolute fluorescence in $80 \%$ of the sampled locations, results from small surface angle changes, and this is generally what should be expected in in vivo applications. The observed variation in absolute fluorescence in the other $20 \%$ of the sampled locations may result from some inhomogeneity introduced by the process of obtaining and handling an ex vivo sample and not the general existence of some form of optical inhomogeneity on the scale of the laser diameter on the surface of spinal cord. Included in this effect would be the fact that the efficiency of observation of emission from beneath the surface also depends on the angle of incidence and the exact depth of a particular fluorophore. ${ }^{33}$ Thus the overall consistency of the data suggests that the procedure of comparing Raman spectra that have been treated with the SNV transform should accurately reflect relative changes in the composition of the probed tissues. ${ }^{13,14}$

Significantly lower fluorescence in injured spinal cords in comparison to healthy spinal cords was observed, indicating that fluorescence by itself can be used as an independent measure of demarcating lesion boundaries from uninjured regions. The absolute fluorescence is important in the context of ex vivo and in vivo studies if there is a desire to potentially associate the fluorescence quantitatively with the volume of the probed region. In order to quantify Raman spectra for biomedical samples in the past we have normalized Raman spectra to the underlying fluorescence with some success ${ }^{17,36}$ but this approach may be problematic for in vivo SCI applications if we opt to completely avoid physical contact between the surface of the cord and any type of optical aperture ${ }^{35}$ or template. This geometry with free space coupling minimizes the production of spurious Raman and fluorescence of the optical components themselves. ${ }^{36}$ It is certainly conceivable that in practice for in vivo applications, monitoring the specular reflection from the cord surface could potentially provide a completely noncontact feedback mechanism for initially setting and then maintaining an optimal or consistent angle of incidence while probing the cord. This 
approach might be easier to accomplish in non-normal incidence and overall this might offer a more consistent basis for comparing absolute fluorescence from location to location, and at the same location, over the passing of time.

Given the observed site-to-site variation for the same cord, the variation of the absolute fluorescence with time post-injury, averaged over multiple animals and locations, is significant. The reduction in fluorescence can be a direct result of the resulting cell death and demyelination that occurs as a result of SCI. It is also possible that normal homeostasis produces more of some fluorescent species per unit volume than injured tissue, and less of at least one other fluorescent species. We recently reported NIR fluorescence and photobleaching effects in human fingertip skin in vivo very reminiscent of the observations in this study. It was found that the advanced glycation end product pentosidine fluoresces in the NIR. ${ }^{20}$

The wider spectral emission in injured cord suggests that there is apparently another significant fluorophore in injured cord that is not present in healthy cord, although the ratio of the two components seems to be constant within all of the injured cord data and within all of the healthy cord data. We hypothesize that a likely new significant fluorophore is trapped dried blood that would presumably contain methemoglobin and should have a fluorescence that is shifted from that of other hemoglobins since the absorption spectrum is shifted. These observations could be connected to the tissues' attempt to revascularize. ${ }^{37}$

Each rat's entire circulatory system was thoroughly perfused with PBS before the cord was removed in an effort to remove all traces of the blood, particularly hemoglobin, from the probed volume. But given the strong fluorescence quantum yield of all porphyrins, we never expected to succeed in completely removing all heme-based emission. Since hemoglobin is generally the strongest fluorophore (per unit volume) in any probed absolute volume in vivo, we hypothesize that the total fluorescence could be a function of the degree to which a probed volume of the SCI has been revascularized. However, there is no unequivocal evidence from the data shown in this paper to suggest that the fluorescence from the injured cords must originate from a blood borne fluorophore.

Since only the gray matter is vascularized and the spinal gray matter is covered by the more highly scattering but unvascularized white matter, the choice of $785 \mathrm{~nm}$ excitation wavelength may have sufficiently precluded efficient depth penetration to produce observable fluorescence. We further observed that some of the Raman features actually do have a nonmonotonic time course post-SCI. In the range of $960-970 \mathrm{~cm}^{-1}$ and near $1560 \mathrm{~cm}^{-1}$ (Heme group) Raman shift, we see a decrease-increase-decrease time course as might correlate with revascularization. Actual resonance and spontaneous Raman features for hemoglobin are known ${ }^{24}$ in these regions but these would then be expected to correlate with the fluorescence behavior if the majority of the observed fluorescence can in fact be associated with incompletely removed blood by the PBS perfusion. There may be other, possibly more productive, fluorophores than hemoglobin in the probed volume. If that is the case, then it is possible that we could observe revascularization using Raman features and not be able to observe a correlated change in observed fluorescence. Further, there is scant data in the literature about the optical properties of glial scars. In order to use fluorescence to qualitatively assess the probed volume, more information is needed about the scattering and absorption properties of injured spinal cords.

\section{Conclusions}

The results of this study demonstrate that injured spinal cords can be readily distinguished from healthy spinal cords in two models of SCI. There are significant differences between the Raman spectra and fluorescence of healthy spinal cord and injured cord. These differences, and their time course post-injury, correlate with demyelination and CSPG and GAG upregulation; experimentally observed tissue and metabolic changes that accompany SCI. Further, the action of cABC on CS6 can be easily discerned and followed in vitro. The Raman spectra of CS6 digestion and difference spectra from injured spinal cords showed distinct features beyond $1670 \mathrm{~cm}^{-1}$ indicating the possible formation of a lactone in vivo since the lactone carbonyl stretch occurs in a region that is unlikely to be obscured by spectral congestion. Thus NIR Raman offers some very exciting possibilities with respect to monitoring SCI and related treatments in vivo in the future.

\section{Acknowledgments}

A fellowship from the Syracuse Biomaterials Institute supported Tarun Saxena. Kyle Hoellger and Karan Shertukde assisted in the collection of data from healthy and contused spinal cords. Machine shop fabrication was by Lou Buda, Charlie Brown, Phil Arnold and Les Schmutzler. Sally Prasch at the glass shop fabricated the cuvettes. Assistance with the design and optimization of our optical system that was provided by LighTouch Medical and by Rebecca J. Bussjager and discussions regarding the neurology in general with Dr. George Shaheen are gratefully acknowledged. Assistance in the implementation of the KNN algorithm by Arun Subramanian is greatly appreciated. Critical reading of the manuscript by Dr. Jeremy Gilbert is greatly appreciated. Assistance in immunohistological preparations by Karen Hughes and Grazyna Rygiel is greatly appreciated. Funding of the Syracuse University REU program made the participation of Kyle Hoellger possible.

\section{References}

1. National Spinal Cord Injury Statistical Center, "Spinal cord injury facts and figures at a glance" (Birmingham, Alabama, 2010), https://www.nscisc.uab.edu/public_content/pdf/Facts\%20and $\% 20$ Figures\%20at\%20a\%20Glance\%202010.pdf.

2. Christopher and Dana Reeve Foundation, "One degree of separation paralysis and spinal cord injury in the United States" (2009), http://www.christopherreeve.org/atf/cf/\%7B3d83418f-b9674c18-8ada-adc2e5355071\%7D/8112REPTFINAL.PDF.

3. J. Silver and J. H. Miller, "Regeneration beyond the glial scar," Nat. Rev. Neurosci. 5, 146-156 (2004).

4. M. M. Siegenthaler, M. K. Tu, and H. S. Keirstead, "The extent of myelin pathology differs following contusion and transection spinal cord injury," J. Neurotrauma 24, 1631-1646 (2007).

5. T. Hagg and M. Oudega, "Degenerative and spontaneous regenerative processes after spinal cord injury," J. Neurotrauma 23, 264-280 (2006).

6. S. M. Onifer, A. G. Rabchevsky, and S. W. Scheff, "Rat models of traumatic spinal cord injury to assess motor recovery," ILAR J. 48(4), 385-395 (2007). 
7. S. Yu and R. V. Bellamkonda, "Dorsal root ganglia neurite extension is inhibited by mechanical and chondroitin sulfate-rich interfaces," $J$. Neurosci. Res. 66, 303-310 (2001).

8. S. Ramóny Cajal, in Degeneration and Regeneration of the Nervous System, Oxford University, London (1928).

9. E. J. Bradbury, L. D. F. Moon, R. J. Popat, V. R. King, G. S. Bennett, P. N. Patel, J. W. Fawcett, and S. B. McMahon, "Chondroitinase ABC promotes functional recovery after spinal cord injury," Nature $\mathbf{4 1 6}$ 636-640 (2002).

10. A. T. Tu, in Raman Spectroscopy in Biology: Principles and Applications, Wiley, New York (1982).

11. S. W. Scheff, A. G. Rabchevsky, I. Fugaccia, J. A. Main, and J. E. Lumpp, Jr., "Experimental modeling of spinal cord injury: characterization of a force-defined injury device," J. Neurotrauma 20, 179-193 (2003).

12. T. Saxena, B. Deng, J. Hasenwinkel, E. Lewis-Clark, K. Hoellger, D. Stelzner, and J. Chaiken, "Near infrared Raman spectroscopic study of reactive gliosis and the glial scar in injured rat spinal cords," Proc. SPIE 7560, 756001 (2010).

13. R. Szostak, "Simple transformation of spectra to effectively reduce quantification errors in FT-Raman multivariate analysis of complex systems," Vib. Spectrosc. 49, 298 (2009).

14. R. J. Barnes, "Standard normal variate transformation and de-trending of near-infrared diffuse reflectance spectra," Appl. Spectrosc. 43, 772 (1989).

15. P. Mosier-Boss, S. Lieberman, and R. Newberry, "Fluorescence Rejection in Raman Spectroscopy by Shifted-Spectra, Edge Detection, and FFT Filtering techniques,” Appl. Spectrosc. 49, 630 (1995).

16. C. A. Lieber and A. Mahadevan-Jansen, "Automated Method for Subtraction of Fluorescence from Biological Raman Spectra," Appl. Spectrosc. 57, 1363-1367 (2003).

17. J. Chaiken and W. Finney, "Effect of hemoglobin concentration variation on the accuracy and precision of glucose analysis using tissue modulated, noninvasive, in vivo Raman spectroscopy of human blood: a small clinical study," J. Biomed. Opt. 10, 031111 (2005).

18. J. Chaiken, J. Goodisman, B. Deng, R. J. Bussjager, and G. Shaheen, "Simultaneous, noninvasive observation of elastic scattering, fluorescence and inelastic scattering as a monitor of blood flow and hematocrit in human fingertip capillary beds," J. Biomed. Opt. 14, 050505 (2009).

19. J. Chaiken, B. Deng, R. J. Bussjager, G. Shaheen, D. Rice, D. Stehlik, and J. Fayos, "Instrument for near infrared emission spectroscopic probing of human fingertips in vivo," Rev. Sci. Instrum. 81, 034301 (2010).

20. B. Deng, C. Wright, E. Lewis-Clark, R. Geier, G. Shaheen, and J. Chaiken, "Direct noninvasive observation of near infrared photobleaching of autofluorescence in human volar side fingertips in vivo," Proc. SPIE 7560, 75600P (2010).

21. R. Bansil, I. V. Yannas, and H. E. Stanley, "Raman spectroscopy: a structural probe of glycosaminoglycans," Biochim. Biophys. Acta 541, 535-542 (1978).

22. N. Amharref, A. Beljebbar, S. Dukic, L. Venteo, L. Schneider, M. Pluot, and M. Manfait, "Discriminating healthy from tumor and necrosis tissue in rat brain tissue samples by Raman spectral imaging," Biochim. Biophys. Acta 1768, 2605-2615 (2007).
23. R. Ellis, E. Green, and C. P. Winlove, "Structural analysis of glycosaminoglycans and proteoglycans by means of Raman microspectrometry," Connect. Tissue Res. 50, 29-36 (2009).

24. A. R. Ishwar, K. J. Jeong, A. Panitch, and O. Akkus, "Raman spectroscopic investigation of peptide-glycosaminoglycan interactions," Appl. Spectrosc. 63, 636-641 (2009).

25. S. Koljenovic, T. B. Schut, A. Vincent, J. M. Kros, and G. J. Puppels, "Detection of meningioma in dura mater by Raman spectroscopy," Anal. Chem. 77, 7958-7965 (2005).

26. S. Franzen, S. E. Wallace-Williams, and A. P. Shreve, "Heme chargetransfer band III is vibronically coupled to the Soret band," J. Am. Chem. Soc. 124, 7146-7155 (2002).

27. L. L. Jones, R. U. Margolis, and M. H. Tuszynski, "The chondroitin sulfate proteoglycans neurocan, brevican, phosphacan, and versican are differentially regulated following spinal cord injury," Exp. Neurol. 182, 399-411 (2003).

28. J. W. Fawcett and R. A. Asher, "The glial scar and central nervous system repair," Brain Res. Bull. 49, 377-391 (1999).

29. J. Struve, P. C. Maher, Y. Q. Li, S. Kinney, M. G. Feblings, C. Kuntz 4th, and L. S. Sherman, "Disruption of the hyaluronan-based extracellular matrix in spinal cord promotes astrocyte proliferation," Glia 52, 16-24 (2005).

30. S. A. Back and T. M. F. Tuohy, "Hyaluronan accumulates in demyelinated lesions and inhibits oligodendrocyte progenitor maturation," Nat. Med. 11, 966-972 (2005).

31. B. K. Kwon and A. M. Stammers, "Cerebrospinal fluid inflammatory cytokines and biomarkers of injury severity in acute human spinal cord injury," J. Neurotrauma 27, 669-682 (2010)

32. R. A. Vaishnav, I. N. Singh, D. M. Miller, and E. D. Hall, "Lipid Peroxidation-Derived Reactive Aldehydes Directly and Differentially Impair Spinal Cord and Brain Mitochondrial Function," J. Neurotrauma 27(7), 1311-1320 (2010).

33. R. P. Bunge, W. R. Puckett, and E. D. Hiester, "Observations on the pathology of several types of human spinal cord injury, with emphasis on the astrocyte response to penetrating injuries," Adv. Neurol. 72, 305315 (1997).

34. C. Iannotti, Y. P. Zhang, L. B. Shields, Y. Han, D. A. Burke, X. M. Xu, and C. B. Shields, "Dural repair reduces connective tissue scar invasion and cystic cavity formation after acute spinal cord laceration injury in adult rats," J. Neurotrauma 23, 853-865 (2006).

35. P. Matousek, M. D. Morris, N. Everall, I. P. Clark, M. Towrie, E. Draper, A. Goodship, and A. W. Parker, "Numerical simulations of subsurface probing in diffusely scattering media using spatially offset Raman spectroscopy," Appl. Spectrosc. 59, 1485-1492 (2005).

36. J. Chaiken, W. F. Finney, C. M. Peterson, K. P. Peterson, P. E. Knudson, R. S. Weinstock, and P. Lein, "Noninvasive, in-vivo, tissue modulated near infrared vibrational spectroscopic study of mobile and static tissues: blood chemistry," Proc. SPIE 3918, 135-143 (2000).

37. N. Weidner, R. J. Grill, and M. H. Tuszynski, "Elimination of basal lamina and the collagen 'scar' after spinal cord injury fails to augment corticospinal tract regeneration," Exp. Neurol. 160, 40-50 (1999). 